\title{
Relation-Changing Logics as Fragments of Hybrid Logics
}

\author{
Carlos Areces \\ Universidad Nacional de Córdoba, \\ Argentina \\ CONICET, Argentina
}

\author{
Raul Fervari \\ Universidad Nacional de Córdoba, \\ Argentina \\ CONICET, Argentina \\ Mauricio Martel \\ Universität Bremen, Germany
}

\author{
Guillaume Hoffmann \\ Universidad Nacional de Córdoba, \\ Argentina \\ CONICET, Argentina
}

\begin{abstract}
Relation-changing modal logics are extensions of the basic modal logic that allow changes to the accessibility relation of a model during the evaluation of a formula. In particular, they are equipped with dynamic modalities that are able to delete, add, and swap edges in the model, both locally and globally. We provide translations from these logics to hybrid logic along with an implementation. In general, these logics are undecidable, but we use our translations to identify decidable fragments. We also compare the expressive power of relation-changing modal logics with hybrid logics.
\end{abstract}

\section{Introduction}

Modal logics $[10,11]$ were originally conceived as logics of necessary and possible truths. They are now viewed, more broadly, as logics that explore a wide range of modalities, or modes of truth: epistemic ("it is known that"), doxastic ("it is believed that"), deontic ("it ought to be the case that"), or temporal ("it has been the case that"), among others. From a model-theoretic perspective, the field evolved into a discipline that deals with languages interpreted on various kinds of relational structures or graphs. Nowadays, modal logics are actively used in areas as diverse as software verification, artificial intelligence, semantics and pragmatics of natural language, law, philosophy, etc.

As we just mentioned, from an abstract point of view, modal logics can be seen as formal languages to navigate and explore properties of a given relational structure. If we are interested, on the other hand, in describing how a given relational structure evolves (through time or through the application of certain operations) then classical modal languages seem a priori to fall short of the mark. Of course, it is always possible to model the whole space of possible transformations as a graph, and use modal languages at that level, but this soon becomes unwieldy. It would be more elegant to use dynamic modalities that mimic the changes the structure will undergo.

There exist several dynamic modal operators that fit in this approach. A clear example are the dynamic operators introduced in dynamic epistemic logics (see, e.g., [15]). These operators are used to model changes in the epistemic state of an agent by removing edges from the graph that represents the information states the agent considers possible. A less obvious example is given by hybrid logics $[2,12]$ equipped with the down arrow operator $\downarrow$ which is used to 'rebind' names for states to the current point of evaluation. Finally, a classical example is Sabotage Logic introduced by van Benthem in [9]. The sabotage operator deletes individual edges in a graph and was introduced to solve the sabotage game. This game is played on a graph by two players, Runner and Blocker. Runner can move on the graph from node to accessible node, starting from a designated point, and with the goal of reaching a given final point. Blocker, on the other hand, can delete one edge from the graph every time it is his turn. Runner wins if he manages to move from the origin to the final point, while Blocker wins otherwise. Van

D. Cantone and G. Delzanno (Eds.): Seventh Symposium on Games, Automata, Logics and Formal Verification (GandALF'16) EPTCS 226, 2016, pp. 16-29, doi:10.4204/EPTCS.226.2 (c) Areces, Fervari, Hoffmann and Martel This work is licensed under the Creative Commons Attribution License. 
Benthem turns the sabotage game into a modal logic, where the (global) sabotage operator $\langle\mathrm{gsb}\rangle$ models the moves of Blocker, and is interpreted on a graph $\mathscr{M}$ at a point $w$ as:

$$
\mathscr{M}, w \models\langle\mathrm{gsb}\rangle \varphi \text { iff there is a pair }(u, v) \text { of } \mathscr{M}_{\text {such }} \text { that } \mathscr{M}_{(u, v)}^{-}, w=\varphi
$$

where $\mathscr{M}_{(u, v)}^{-}$is identical to $\mathscr{M}$ except that the edge $(u, v)$ has been removed. The moves of Runner, on the other hand, can be modeled using the standard $\diamond$ operator of classical modal logics.

More recently, Sabotage Logic was proposed as a formalism for reasoning about formal learning theory [18]. Learning can be seen as a game with two players, Teacher and Learner, where Learner changes his information state through a step-by-step process. The process is successful if he eventually reaches an information state describing the real state of affairs. The information that Teacher provides can be interpreted as feedback about Learner's conjectures about the current state of affairs, allowing him to discard inconsistent hypotheses. It should be clear that from this game-theoretical perspective, the interaction between Teacher and Learner can be modeled using Sabotage Logic.

The dynamic approach seems appealing and very flexible: it is easy to come up with situations that nicely fit and extend the examples we just mentioned. Discovering alternative routes for Runner in van Benthem's sabotage game, or possible shortcuts that Learner can take in learning theory can be modeled by adding new edges to the graph. Swapping an edge can be used to represent other scenarios such as changing the direction of a route, or allowing Learner to return to a previous information state. All these primitives can also be turned into a modal logic in the same way as Sabotage Logic, in order to get a formal language for reasoning about the games.

Motivated by scenarios like the ones we just described, we investigate three dynamic primitives that can change the accessibility relation of a model: sabotage (deletes edges from the model), bridge (adds edges to a model), and swap (turns around edges), both in a global version (performing changes anywhere in the model) and local (changing adjacent edges from the evaluation point). We have chosen these relation-changing operators with the intention of covering a sufficiently varied sample of alternatives, as it is done in previous works. In [5] we first introduced the primitives, and we investigated their expressive power and model checking problem. We introduced tableaux methods for relation-changing modal logics in [6]. In [7] we studied local swap logic, in particular its decidability problem and its relation with first-order and hybrid logics. In [8] a general framework for representing model updates is defined, and connections with dynamic epistemic logic were introduced in $[3,4]$. Finally, we know that the satisfiability problem for the six relation-changing logics considered is undecidable $[21,16,7,22]$.

In this article, we show that relation-changing logics can be seen as fragments of hybrid logics. We consider hybrid logics because it is the best known modal logic that can simulate the semantics of relation-changing operators. We introduce translations to $\mathscr{H} \mathscr{L}(\mathrm{E}, \downarrow)$, the basic modal logic extended with nominals, the down arrow binder $\downarrow$, and the universal modality $\mathrm{E}$ (in some cases the translations fall into the less expressive hybrid logic $\mathscr{H} \mathscr{L}(:, \downarrow)$, i.e., with the satisfiability operator : instead of E). We also show that relation-changing logics are strictly less expressive than the hybrid logics they are translated into. Then, we discuss how we can benefit from known decidable fragments of $\mathscr{H} \mathscr{L}(\mathrm{E}, \downarrow)$ to find decidable fragments of relation-changing modal logics. Finally, we have implemented these translations into the hybrid logic prover HTab [20] so that it can now reason on relation-changing formulas.

The article is organized as follows. In Section 2 we introduce the syntax and semantics of relationchanging modal logics. Section 3 introduces different hybrid extensions of modal logic which are used in Section 4 to encode relation-changing operators. An implementation is described in Section 5 with some examples. Then, in Section 6 we discuss decidability results, and in Section 7 we compare the expressivity of relation-changing logics and hybrid logics. Finally, we conclude with some remarks and future work in Section 8. 


\section{Relation-Changing Modal Logics}

In this section, we formally introduce extensions of the basic modal logic with relation-changing operators. For more details, we direct the reader to, e.g., [16].

Definition 1 (Syntax). Let PROP be a countable, infinite set of propositional symbols. The set FORM of formulas over PROP is defined as:

$$
\text { FORM ::= }=\perp p|\neg \varphi| \varphi \wedge \psi|\diamond \varphi| \diamond \varphi,
$$

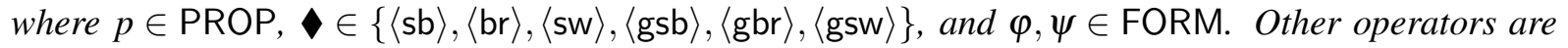
defined as usual.

Let $\mathscr{M} \mathscr{L}$ (the basic modal logic) be the logic without the $\{\langle\mathrm{sb}\rangle,\langle\mathrm{br}\rangle,\langle\mathrm{sw}\rangle,\langle\mathrm{gsb}\rangle,\langle\mathrm{gbr}\rangle,\langle\mathrm{gsw}\rangle\}$ oper-

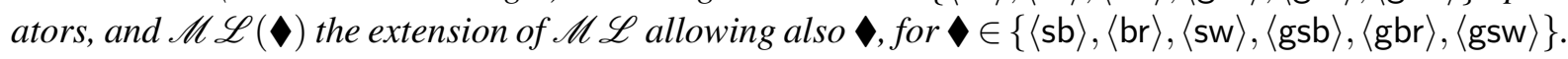
In particular, $\mathscr{M} \mathscr{L}(\langle\mathrm{sb}\rangle,\langle\mathrm{gsb}\rangle), \mathscr{M} \mathscr{L}(\langle\mathrm{br}\rangle,\langle\mathrm{gbr}\rangle)$, and $\mathscr{M} \mathscr{L}(\langle\mathrm{sw}\rangle,\langle\mathrm{gsw}\rangle)$ will be called Sabotage Logic, Bridge Logic, and Swap Logic, respectively.

Semantically, formulas are evaluated in standard relational models, and the meaning of the operators of the basic modal logic remains unchanged (see [11] for details). When we evaluate formulas containing relation-changing operators, we will need to keep track of the edges that have been modified. To that end, let us define precisely the models that we will use.

Definition 2 (Models and model updates). A model $\mathscr{M}$ is a triple $\mathscr{M}=\langle W, R, V\rangle$, where $W$ is a nonempty set whose elements are called points or states; $R \subseteq W \times W$ is the accessibility relation; and $V$ : $\mathrm{PROP} \rightarrow \mathscr{P}(W)$ is a valuation. We define the following notations:

$$
\begin{array}{ll}
\text { (sabotaging) } & \mathscr{M}_{S}^{-}=\left\langle W, R_{S}^{-}, V\right\rangle \text {, with } R_{S}^{-}=R \backslash S, S \subseteq R . \\
\text { (bridging) } & \mathscr{M}_{S}^{+}=\left\langle W, R_{S}^{+}, V\right\rangle \text {, with } R_{S}^{+}=R \cup S, S \subseteq(W \times W) \backslash R \text {. } \\
\text { (swapping) } & \mathscr{M}_{S}^{*}=\left\langle W, R_{S}^{*}, V\right\rangle \text {, with } R_{S}^{*}=\left(R \backslash S^{-1}\right) \cup S, S \subseteq R^{-1} .
\end{array}
$$

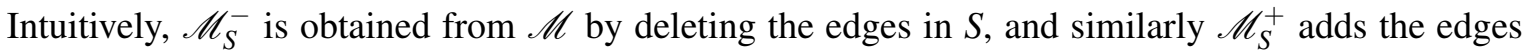
in $S$ to the accessibility relation, and $\mathscr{M}_{S}^{*}$ adds the edges in $S$ as inverses of edges previously in the accessibility relation.

Let $w$ be a state in $\mathscr{M}$, the pair $(\mathscr{M}, w)$ is called a pointed model; we will usually drop parenthesis and write $\mathscr{M}, w$ instead of $(\mathscr{M}, w)$. In the rest of this article, we will use $w v$ as a shorthand for $\{(w, v)\}$ or $(w, v)$; context will always disambiguate the intended use.

Definition 3 (Semantics). Given a pointed model $\mathscr{M}, w$ and a formula $\varphi$, we say that $\mathscr{M}, w$ satisfies $\varphi$, and write $\mathscr{M}, w \models \varphi$, when

$$
\begin{aligned}
& \mathscr{M}, w=p \quad \text { iff } \quad w \in V(p) \\
& \mathscr{M}, w=\neg \varphi \quad \text { iff } \quad \mathscr{M}, w \not=\varphi \\
& \mathscr{M}, w=\varphi \wedge \psi \quad \text { iff } \quad \mathscr{M}, w=\varphi \text { and } \mathscr{M}, w=\psi \\
& \mathscr{M}, w=\diamond \varphi \quad \text { iff } \quad \text { for some } v \in W \text { s.t. }(w, v) \in R, \mathscr{M}, v \models \varphi \\
& \mathscr{M}, w=\langle\mathrm{sb}\rangle \varphi \quad \text { iff } \quad \text { for some } v \in W \text { s.t. }(w, v) \in R, \mathscr{M}_{w v}^{-}, v=\varphi \\
& \mathscr{M}, w=\langle\mathrm{br}\rangle \varphi \quad \text { iff } \quad \text { for some } v \in W \text { s.t. }(w, v) \notin R, \mathscr{M}_{w v}^{+}, v=\varphi \\
& \mathscr{M}, w=\langle\mathrm{sw}\rangle \varphi \quad \text { iff } \quad \text { for some } v \in W \text { s.t. }(w, v) \in R, \mathscr{M}_{v w}^{*}, v=\varphi \\
& \mathscr{M}, w=\langle\mathrm{gsb}\rangle \varphi \quad \text { iff } \quad \text { for some } v, u \in W \text {, s.t. }(v, u) \in R, \mathscr{M}_{v u}^{-}, w=\varphi \\
& \mathscr{M}, w=\langle\text { gbr }\rangle \varphi \quad \text { iff } \quad \text { for some } v, u \in W \text {, s.t. }(v, u) \notin R, \mathscr{M}_{v u}^{+}, w=\varphi \\
& \mathscr{M}, w=\langle\mathrm{gsw}\rangle \varphi \quad \text { iff } \quad \text { for some } v, u \in W \text {, s.t. }(v, u) \in R, \mathscr{M}_{u v}^{*}, w \models \varphi \text {. }
\end{aligned}
$$

We say that $\varphi$ is satisfiable if for some pointed model $\mathscr{M}, w$ we have $\mathscr{M}, w=\varphi$. 
The meaning of the relation-changing operators $\langle\mathrm{sb}\rangle$ (local sabotage), $\langle\mathrm{br}\rangle$ (local bridge), $\langle\mathrm{sw}\rangle$ (local swap), 〈gsb $\rangle$ (global sabotage), 〈gbr $\rangle$ (global bridge) and $\langle g s w\rangle$ (global swap) should be clear from the semantic conditions above. The local operators alter one arrow which is adjacent to the point of evaluation (deleting, adding and swapping it, respectively) while the global versions can change an arrow anywhere in the model.

\section{Extensions of Modal Logic and Hybrid Logic}

In this section, we present several extensions of the basic modal logic $\mathscr{M} \mathscr{L}$. The existential modality [19], written $\mathrm{E} \varphi$, extends $\mathscr{M} \mathscr{L}$ in the following way:

$$
\mathscr{M}, w=\mathrm{E} \varphi \quad \text { iff } \quad \text { for some } v \in W, \mathscr{M}, v \models \varphi .
$$

In words, $\mathrm{E} \varphi$ is true at a state $w$ if $\varphi$ is true somewhere in the model. The $\mathrm{E}$ operator, with its dual $\mathrm{A}$, has been extensively investigated in classical modal logic [25].

Now we consider several traditional 'hybrid' operators (see [2] for details): nominals, the satisfaction operator, and the down-arrow binder. The basic hybrid logic $\mathscr{H} \mathscr{L}$ is obtained by adding nominals to $\mathscr{M} \mathscr{L}$. A nominal is a propositional symbol that is true at exactly one state in a model. Fix the signature $\langle$ PROP, NOM〉, with NOM $\subseteq$ PROP. For $n \in$ NOM, we require that its valuation is a singleton set, i.e., there is a single state $w$ such that $V(n)=\{w\}$. In addition to nominals, hybrid logic typically involves the satisfaction operator. Given a nominal $n$ and a formula $\varphi$, the satisfaction operator is written $n: \varphi$. The intended meaning is " $\varphi$ is true at the state named by $n$ ". Its semantics is given by the following clause:

$$
\mathscr{M}, w=n: \varphi \quad \text { iff } \quad \mathscr{M}, v \models \varphi \text { where } V(n)=\{v\} .
$$

Observe that if the language has the $\mathrm{E}$ operator and nominals, then $n: \varphi$ is definable because $n: \varphi$ is equivalent to $\mathrm{E}(n \wedge \varphi)$.

Finally, consider the down-arrow binder operator, written $\downarrow$. Let the valuation $V_{n}^{w}$ be defined by $V_{n}^{w}(n)=\{w\}$ and $V_{n}^{w}(m)=V(m)$, when $n \neq m$. The semantic condition for $\downarrow$ is the following:

$$
\langle W, R, V\rangle, w=\downarrow n . \varphi \quad \text { iff } \quad\left\langle W, R, V_{n}^{w}\right\rangle, w=\varphi .
$$

The language $\mathscr{H} \mathscr{L}(:, \downarrow)$ is a reduction class of first-order logic, and is thus undecidable [12, 13]. It remains undecidable even with a single accessibility relation, no satisfaction operator, and only nominal propositional symbols [1]. $\mathscr{H} \mathscr{L}(\mathrm{E}, \downarrow)$ is equivalent to first-order logic, since $\downarrow$ can define the operators $\exists$ and $\forall$ when combined with $E$ and $A$.

Contrary to relation-changing modal logics, the logic $\mathscr{H} \mathscr{L}(\mathrm{E}, \downarrow)$ is not able to modify the accessibility relation of a model. However, it can use the binder to name as many states as needed. Being able to name states implies that we can also name specific edges in the model. This is what the translations introduced in the next section will exploit.

\section{Translations to Hybrid Logics}

Relation-changing (RC) logics and hybrid logics with the binder $\downarrow$ are two families of logics that are dynamic in their own way. The dynamicity of RC logics is quite obvious: they are able to modify the accessibility relation in a model in an explicit way. On the other hand, hybrid logics carefully move 
nominals around, avoiding to touch anything else in the model. If we consider both formalisms, it would seem that hybrid logics are the gentler and weaker of both. However, this is not true. Hybrid logics have the advantage of surgical precision over RC logics. Being able to name states of the model and use these names turns out to be a crucial advantage. As we will see now, naming can be used to manipulate edges by naming pairs of states using the pattern $\downarrow x$. $\diamond \downarrow y$. $\varphi$. We use this naming technique to simulate edge deletion, addition, and swapping.

Our translations are parametrized over a set of pair of nominals $S \subseteq \mathrm{NOM} \times \mathrm{NOM}$. For a given RC formula $\varphi$, we write its translation as a hybrid formula $(\varphi)_{S}^{\prime}$. When translating a formula, $S$ will originally be empty and it will store pairs of nominals that we will use to simulate the edges affected by the relation-changing operators we encounter during the translation.

Intuitively, given that the hybrid operators cannot affect the accessibility relation, we have to simulate the updates by recording possible affected edges using nominals and $\downarrow$. Notice that as a result, in all the relation-changing logics we will consider, the RC formula $\diamond \psi$ cannot be simply translated into a hybrid formula $\diamond(\psi)_{S}^{\prime}$, even though we have $\diamond$ at our disposition in the hybrid language, because in the source language $\diamond$ is interpreted over the updated accessibility relation. Instead, diamond-formulas need to be translated in a way that takes into account the edges that should be considered deleted, added, or swapped. This is why the translation of diamond-formulas involve the $\diamond$ operator mixed with specific considerations about the set of altered edges $S$.

Consider Sabotage Logic with either the local or global operator. We use the set $S \subseteq \mathrm{NOM} \times \mathrm{NOM}$ to represent sabotaged edges, i.e., edges that have been deleted in a given updated model.

Definition 4 (Sabotage to Hybrid Logic). Let $S \subseteq$ NOM $\times$ NOM and $n \in$ NOM. We define the translation ()$_{S}^{\prime}$ from formulas of $\mathscr{M} \mathscr{L}(\langle\mathrm{sb}\rangle,\langle\mathrm{gsb}\rangle)$ to formulas of $\mathscr{H} \mathscr{L}(\mathrm{E}, \downarrow)$ as:

$$
\begin{aligned}
& (p)_{S}^{\prime}=p \\
& (\neg \varphi)_{S}^{\prime}=\neg(\varphi)_{S}^{\prime} \\
& (\varphi \wedge \psi)_{S}^{\prime}=(\varphi)_{S}^{\prime} \wedge(\psi)_{S}^{\prime} \\
& (\diamond \varphi)_{S}^{\prime}=\downarrow n . \diamond\left(\neg \text { belongs }(n, S) \wedge(\varphi)_{S}^{\prime}\right) \\
& (\langle\mathrm{sb}\rangle \varphi)_{S}^{\prime}=\downarrow n \cdot \diamond\left(\neg \operatorname{belongs}(n, S) \wedge \downarrow m .(\varphi)_{S \cup n m}^{\prime}\right) \\
& (\langle\mathrm{gsb}\rangle \varphi)_{S}^{\prime}=\downarrow k . \mathrm{E} \downarrow n . \diamond\left(\neg \operatorname{belongs}(n, S) \wedge \downarrow m . k:(\varphi)_{S \cup n m}^{\prime}\right)
\end{aligned}
$$

where $n, m$ and $k$ are nominals that do not appear in $S$, and:

$$
\operatorname{belongs}(n, S)=\bigvee_{x y \in S}(y \wedge n: x)
$$

A few comments are in order to understand the translation. First, given some model $\mathscr{M}=\langle W, R, V\rangle$ and some set $S \subseteq \mathrm{NOM} \times \mathrm{NOM}$, the formula $\downarrow n . \vee(\neg$ belongs $(n, S))$ is true at some state $w \in W$ if there exists some state $v$ such that $(w, v) \in R$ and there is no pair of nominals $(x, y) \in S$ such that $(V(x), V(y))=$ $(w, v)$. Then, observe that the cases for $\langle\mathrm{sb}\rangle$ and $\langle\mathrm{gsb}\rangle$ modify the set of deleted pairs in the recursive call to the translation, in both cases by adding an edge named $\mathrm{nm}$. In the $\langle\mathrm{sb}\rangle$ case, $n$ names the evaluation state of the formula, while in the $\langle\mathrm{gsb}\rangle$ case, $n$ names some state anywhere in the model.

Finally, all nominals introduced by the translation are bounded exactly once. Then we can define the following unequivocal notation: let $S \subseteq \mathrm{NOM} \times \operatorname{NOM}$, we define $\bar{S}=\{(\bar{x}, \bar{y}) \mid(x, y) \in S\}$, where $\bar{n}$ is the state named by the nominal $n \in$ NOM under the current valuation of a model.

When considering the translated formula $(\varphi)_{S}^{\prime}$ and its truth in some model $\mathscr{M}=\langle W, R, V\rangle$, one question that may arise is what should be the initial valuation of the nominals that appear in $(\varphi)_{S}^{\prime}$. By definition of models for hybrid logics, nominals must be true at some state. This is not problematic: in 
$(\varphi)_{S}^{\prime}$, nominals are immediately bounded by the $\downarrow$ operator, so the truth value of $(\varphi)_{S}^{\prime}$ does not depend on their initial valuation. Hence, we can choose some state $w \in W$ and say that all nominals are bounded to it. This enables us to talk about equivalence preservation of the translation: the same model $\mathscr{M}$ can be used for $\varphi$ and its translation $(\varphi)_{S}^{\prime}$ modulo the addition of the set of nominals that appear in $(\varphi)_{S}^{\prime}$ and their valuation to some arbitrary state. Then, we can state:

Theorem 1. For $\mathscr{M}=\langle W, R, V\rangle$ a model, $w \in W$, and $\varphi \in \mathscr{M} \mathscr{L}(\langle\mathrm{sb}\rangle,\langle\mathrm{gsb}\rangle)$ we have:

$$
\mathscr{M}, w=\varphi \text { iff } \mathscr{M}, w \models(\varphi)_{\emptyset}^{\prime} .
$$

Proof. We use structural induction on the relation-changing formula, the inductive hypothesis being:

$$
\mathscr{M}_{\bar{S}}^{-}, w \models \varphi \text { iff }\left\langle W, R, V^{\prime}\right\rangle, w \models(\varphi)_{S}^{\prime}
$$

with $S \subseteq \mathrm{NOM} \times \mathrm{NOM}$, and $V^{\prime}$ is exactly as $V$ except that for all $(x, y) \in S$, there are $v, u \in W$ such that $V^{\prime}(x)=v$ and $V^{\prime}(y)=u$. Boolean cases are straightforward, so we only prove the non-trivial inductive cases.

$\varphi=\diamond \psi:$ For the left to right direction, suppose $\mathscr{M}_{\bar{S}}^{-}, w \models \diamond \psi$. Then there is some $v \in W$ such that $(w, v) \in R_{\bar{S}}^{-}$and $\mathscr{M}_{\bar{S}}^{-}, v \models \psi$. Because $(w, v) \notin \bar{S}$, then there is no $(x, y) \in S$ such that $(\bar{x}, \bar{y})=(w, v)$. By inductive hypothesis, we have $\mathscr{M}, v \models(\psi)_{S}^{\prime}$, and because we can name $w$ with a fresh nominal $n$, we obtain $\left\langle W, R, V_{n}^{w}\right\rangle, v=\neg$ belongs $(n, S) \wedge(\psi)_{S}^{\prime}$. Therefore, we have $\mathscr{M}, w=\downarrow n . \diamond\left(\neg \operatorname{belongs}(n, S) \wedge(\psi)_{S}^{\prime}\right)$, and as a consequence we get $\mathscr{M}, w \models(\psi)_{S}^{\prime}$.

For the other direction, suppose $\mathscr{M}, w \models(\psi)_{S}^{\prime}$, i.e., $\mathscr{M}, w \models \downarrow n . \diamond\left(\neg \operatorname{belongs}(n, S) \wedge(\psi)_{S}^{\prime}\right)$. Then we have $\left\langle W, R, V_{n}^{w}\right\rangle, w \models \diamond\left(\neg\right.$ belongs $\left.(n, S) \wedge(\psi)_{S}^{\prime}\right)$, and, by definition, there is some $v \in W$ such that $(w, v) \in R,\left\langle W, R, V_{n}^{w}\right\rangle, v \models \neg$ belongs $(n, S)$ and $\left\langle W, R, V_{n}^{w}\right\rangle, v \models(\psi)_{S}^{\prime}$. Because we have $\neg$ belongs $(n, S)$, there is no $(x, y) \in S$ such that $(\bar{x}, \bar{y})=(w, v)$, which implies $(w, v) \in R$ if and only if $(w, v) \in R_{\bar{S}}^{-}$. On the other hand, by inductive hypothesis we have $\mathscr{M}_{\bar{S}}^{-}, v=\psi$, then we have $\mathscr{M}_{\bar{S}}^{-}, w=\diamond \psi$.

$\varphi=\langle\mathrm{sb}\rangle \psi$ : For the left to right direction, suppose $\mathscr{M}_{\bar{S}}^{-}, w \mid=\langle\mathrm{sb}\rangle \psi$. Then there is some $v \in W$ such that $(w, v) \in R_{\bar{S}}^{-}$and $\left(\mathscr{M}_{\bar{S}}^{-}\right)_{w v}^{-}, v \models \psi$. This is equivalent to say $\mathscr{M}_{\bar{S} \cup w v}^{-}, v \models \psi$. Because $(w, v) \notin \bar{S}$, then there is no $(x, y) \in S$ such that $(\bar{x}, \bar{y})=(w, v)(\otimes)$. By inductive hypothesis we have $\left\langle W, R,\left(\left(V^{\prime}\right)_{n}^{w}\right)_{m}^{v}\right\rangle, v \models$ $(\psi)_{S \cup n m}^{\prime}$, where $V^{\prime}$ is exactly as $V$ but it binds all the nominals which appear in $S$. By definition, we get $\left.\left\langle W, R,\left(V^{\prime}\right)_{n}^{w}\right)\right\rangle, v \models \downarrow m .(\psi)_{S \cup n m}^{\prime}$, and by $(\otimes)$ we have $\left.\left\langle W, R,\left(V^{\prime}\right)_{n}^{w}\right)\right\rangle, v \models \neg \operatorname{belongs}(n, S) \wedge \downarrow m .(\psi)_{S \cup n m}^{\prime}$. Then (by definition) $\left\langle W, R, V^{\prime}\right\rangle, v \models \downarrow n . \diamond\left(\neg \operatorname{belongs}(n, S) \wedge \downarrow m .(\psi)_{S \cup n m}^{\prime}\right)$, and, as a consequence, we have $\left\langle W, R, V^{\prime}\right\rangle, v \models(\varphi)_{S}^{\prime}$.

For the other direction, suppose $\left\langle W, R, V^{\prime}\right\rangle, w=(\psi)_{S}^{\prime}$, i.e., $\left\langle W, R, V^{\prime}\right\rangle, w=\downarrow n . \diamond(\neg \operatorname{belongs}(n, S) \wedge$ $\downarrow m$. $\left.(\psi)_{S \cup n m}^{\prime}\right)$, where $V^{\prime}$ is exactly as $V$ but it binds all the nominals which appear in $S$. Then, we have $\left\langle W, R,\left(V^{\prime}\right)_{n}^{w}\right\rangle, w=\diamond\left(\neg \operatorname{belongs}(n, S) \wedge \downarrow m\right.$. $\left.(\psi)_{S \cup n m}^{\prime}\right)$, and, by definition, there is some $v \in W$ such that $(w, v) \in R,\left\langle W, R, V_{n}^{w}\right\rangle, v \models \neg$ belongs $(n, S)$ and $\left\langle W, R, V_{n}^{w}\right\rangle, v \models \downarrow m .(\psi)_{S \cup n m}^{\prime}$. Then, $\left\langle W, R,\left(\left(V^{\prime}\right)_{n}^{w}\right)_{m}^{v}\right\rangle, v \models$ $(\psi)_{S \cup n m}^{\prime}$. Because we have $\neg$ belongs $(n, S)$, there is no $(x, y) \in S$ such that $(\bar{x}, \bar{y})=(w, v)$, which implies $(w, v) \in R$ if and only if $(w, v) \in R_{\bar{S}}^{-}$. On the other hand, by inductive hypothesis we have $\mathscr{M}_{\bar{S} \cup w v}^{-}, v \models \psi$, and thus we have $\mathscr{M}_{\bar{S}}^{-}, w=\langle\mathrm{sb}\rangle \psi$.

$\varphi=\langle g s b\rangle \psi$ : this case is very similar to the previous one.

For Bridge Logic, we use the set $B \subseteq \mathrm{NOM} \times \mathrm{NOM}$ to represent the new edges. New edges present in $B$ mean that the translation of the modality $\diamond$ should be able to take them. This explains why the translation of $\diamond$ does not look like a $\diamond$ with an extra condition, but like an $E$ with two possibilities: we traverse an edge that is either in the original model or an edge from the $B$ set. 
Definition 5 (Bridge to Hybrid Logic). Let $B \subseteq \mathrm{NOM} \times \mathrm{NOM}$. We define ()$_{B}^{\prime}$ from formulas of $\mathscr{M} \mathscr{L}(\langle\mathrm{br}\rangle,\langle\mathrm{gbr}\rangle)$ to formulas of $\mathscr{H} \mathscr{L}(\mathrm{E}, \downarrow)$ as:

$$
\begin{aligned}
(p)_{B}^{\prime} & =p \\
(\neg \varphi)_{B}^{\prime} & =\neg(\varphi)_{B}^{\prime} \\
(\varphi \wedge \psi)_{B}^{\prime} & =(\varphi)_{B}^{\prime} \wedge(\psi)_{B}^{\prime} \\
(\triangleright \varphi)_{B}^{\prime} & =\downarrow n . \mathrm{E} \downarrow m\left((n: \diamond m \vee \text { belongs }(n, B)) \wedge(\varphi)_{B}^{\prime}\right) \\
(\langle\mathrm{br}\rangle \varphi)_{B}^{\prime} & =\downarrow n . \mathrm{E} \downarrow m .\left(\neg n: \diamond m \wedge \neg \operatorname{belongs}(n, B) \wedge(\varphi)_{B \cup n m}^{\prime}\right) \\
(\langle\mathrm{gbr}\rangle \varphi)_{B}^{\prime} & =\downarrow k . \mathrm{E} \downarrow n . \mathrm{E} \downarrow m .\left(\neg n: \diamond m \wedge \neg \operatorname{belongs}(n, B) \wedge k:(\varphi)_{B \cup n m}^{\prime}\right)
\end{aligned}
$$

where $n, m$ and $k$ are nominals that do not appear in $B$, and belongs is defined as in Definition 4.

Theorem 2. For $\mathscr{M}=\langle W, R, V\rangle$ a model, $w \in W$, and $\varphi \in \mathscr{M} \mathscr{L}(\langle\mathrm{br}\rangle,\langle\mathrm{gbr}\rangle)$, we have:

$$
\mathscr{M}, w \models \varphi \text { iff } \mathscr{M}, w \models(\varphi)_{\emptyset}^{\prime}
$$

Proof. A similar reasoning can be done with the following inductive hypothesis:

$$
\mathscr{M}_{\bar{B}}^{+}, w \models \varphi \text { iff }\left\langle W, R, V^{\prime}\right\rangle, w \models(\varphi)_{B}^{\prime}
$$

with $B \subseteq \mathrm{NOM} \times \mathrm{NOM}$, and $V^{\prime}$ is exactly as $V$ except that for all $(x, y) \in B$, there are $v, u \in W$ such that $V^{\prime}(x)=v$ and $V^{\prime}(y)=u$.

We finish with the case of Swap Logic. We presented a different translation in [7] for the local case only. As we did for Sabotage Logic, we use $S \subseteq \mathrm{NOM} \times \mathrm{NOM}$ to represent the set of deleted edges, i.e., the edges that should not be possible to traverse in a given updated model. Indeed, swapping a non-reflexive edge of a model has the effect of deleting it, along with adding its inverse. This implies that $S^{-1}$ is a set of edges that we can currently traverse. All of this requires that $S$ do not contain any reflexive edge, since a swapped reflexive edge is not deleted. Neither can it contain a pair of symmetric edges since that would be contradictory.

To ensure this, the translation gets more cautious when handling $\langle\mathrm{sw}\rangle$ and $\langle\mathrm{gsw}\rangle$. When swapping occurs, three possible cases are taken into account. The first one is when a reflexive edge is swapped. In that case, the translation continues with the set $S$ left unchanged, but we require some reflexive edge to be present, be it at the current state for $\langle\mathrm{sw}\rangle$ with $\downarrow n$. $\diamond n$, or anywhere in the model for $\langle g s w\rangle$ with $\mathrm{E} \downarrow n . \diamond n$.

The second case is when we swap an irreflexive edge that has never been swapped before. Hence we ensure that this edge is present in the model, that it is irreflexive, and that neither this edge nor its inverse is in $S$. We then add the nominals that name it to $S$ before moving on with the translation.

The last case is when we traverse an already swapped edge. That is, for some $x y \in S$, we traverse the edge referred to by the nominals $y x$. In this case, we do not need to require the presence of any new edge in the model. We assume to be standing at the state named by $y$ and that the rest of the formula is satisfied at $x$, with the modification that we remove $x y$ from $S$ and add $y x$ to it.

An attentive reader would object: why not just remove $x y$ from the set $S$ since swapping some edge twice just makes it return to its configuration in the original model? The answer is that there is a corner case when some edge and its symmetric are both present in the initial model. Then, the action of swapping it twice is not supposed to restore its symmetric. This is what we do by adding $y x$ to the set $S$ : we ensure the former symmetric edge is no longer present. 
Definition 6 (Swap to Hybrid Logic). Let $S \subseteq \mathrm{NOM} \times \mathrm{NOM}$. We define ()$_{S}^{\prime}$ from formulas of $\mathscr{M} \mathscr{L}(\langle\mathrm{sw}\rangle,\langle\mathrm{gsw}\rangle)$ to formulas of $\mathscr{H} \mathscr{L}(\mathrm{E}, \downarrow)$ as:

$$
\begin{aligned}
(p)_{S}^{\prime}= & p \\
(\neg \varphi)_{S}^{\prime}= & \neg(\varphi)_{S}^{\prime} \\
(\varphi \wedge \psi)_{S}^{\prime}= & (\varphi)_{S}^{\prime} \wedge(\psi)_{S}^{\prime} \\
(\diamond \varphi)_{S}^{\prime}= & \left(\downarrow n \cdot \diamond\left(\neg \text { belongs }(n, S) \wedge(\varphi)_{S}^{\prime}\right)\right) \vee \text { isSat }\left(S^{-1},(\varphi)_{S}^{\prime}\right) \\
(\langle\mathrm{sw}\rangle \varphi)_{S}^{\prime}= & \left(\downarrow n \cdot \diamond n \wedge(\varphi)_{S}^{\prime}\right) \\
& \vee \downarrow n \cdot \diamond\left(\neg n \wedge \neg \text { belongs }(n, S) \wedge \neg \operatorname{belongs}\left(n, S^{-1}\right) \wedge \downarrow m .(\varphi)_{S \cup n m}^{\prime}\right) \\
& \vee \bigvee_{x y \in S}\left(y \wedge x:(\varphi)_{(S \backslash x y) \cup y x}^{\prime}\right) \\
(\langle\mathrm{gsw}\rangle \varphi)_{S}^{\prime}= & \left(\mathrm{E} \downarrow n \cdot \diamond n \wedge(\varphi)_{S}^{\prime}\right) \\
& \vee \downarrow k \cdot \mathrm{E} \downarrow n \cdot \diamond\left(\neg n \wedge \neg \text { belongs }(n, S) \wedge \neg \text { belongs }\left(n, S^{-1}\right) \wedge \downarrow m \cdot k:(\varphi)_{S \cup n m}^{\prime}\right) \\
& \vee \bigvee_{x y \in S}(\varphi)_{(S \backslash x y) \cup y x}^{\prime}
\end{aligned}
$$

where $n, m$ and $k$ are nominals that do not appear in $S$, belongs is defined as in Definition 4 , and

$$
\operatorname{isSat}(S, \varphi)=\bigvee_{x y \in S}(x \wedge y: \varphi)
$$

The formula is $\operatorname{Sat}\left(S,(\varphi)_{S}^{\prime}\right)$ says that the translation of $\varphi$ is satisfiable at the end of some of the edges belonging to $S$. Note that the translation maps formulas of $\mathscr{M} \mathscr{L}(\langle s w\rangle)$ to the less expressive $\mathscr{H} \mathscr{L}(:, \downarrow)$, i.e., the $\mathrm{E}$ operator is not required.

Theorem 3. For $\mathscr{M}=\langle W, R, V\rangle$ a model, $w \in W$ and $\varphi \in \mathscr{M} \mathscr{L}(\langle\mathrm{sw}\rangle,\langle\mathrm{gsw}\rangle)$ we have:

$$
\mathscr{M}, w=\varphi \text { iff } \mathscr{M}, w \models(\varphi)_{\emptyset}^{\prime}
$$

Proof. Again, a similar reasoning can be done with the following inductive hypothesis:

$$
\mathscr{M}_{S^{-1}}^{*}, w \models \varphi \text { iff }\left\langle W, R, V^{\prime}\right\rangle, w=(\varphi)_{S}^{\prime}
$$

with $S \subseteq \mathrm{NOM} \times \mathrm{NOM}$, and $V^{\prime}$ is exactly as $V$ except that for all $(x, y) \in S$, there are $v, u \in W$ such that $V^{\prime}(x)=v$ and $V^{\prime}(y)=u$.

\section{Implementation and Examples}

We have implemented these translations as a new feature of the tableaux-based theorem prover HTab [20]. Its version 1.7.1 can be downloaded from http://hub. darcs . net/gh/htab along with example formulas. Instructions are provided, explaining how to use HTab to check satisfiability of some relationchanging formula and to generate a model from an open tableau.

HTab originally handles the hybrid logic $\mathscr{H} \mathscr{L}(:, \mathrm{E}, \downarrow)$, and guarantees termination of any fragment that lacks the $\downarrow$ binder. As part of the work presented in this article, we added the following feature: when passed the --translate flag, HTab interprets the input formula as a relation-changing one. It first translates it to the corresponding $\mathscr{H} \mathscr{L}(\mathrm{E}, \downarrow)$ formula (or more precisely, $\mathscr{H} \mathscr{L}(:, \downarrow)$ formula in the case of local sabotage and local swap), and then runs its internal hybrid tableaux calculus on the translation.

Since the translation uses the $\downarrow$ binder, HTab may never terminate on some specific relation-changing formulas. Even in the terminating cases, the size of the translated formula (in particular for swap logic) 
can make HTab run for a very long time before giving an answer. However, there are several possible workarounds. First, a time limit in seconds can be given with the flag -t. Also, it may be useful to disable the semantic branching optimization by passing --sembranch=no. Tweaking unit propagation can also be useful for certain formulas, in some cases by disabling it (with --no-unit-prop), and in others, by making it more aggressive (with --eager). The flag --minimal makes HTab try reusing existing states in the model instead of systematically generating new ones. This introduces many more branches in the tableau, making it slower, but it can be crucial to make some cases terminate. Finally, the flag --random uses pseudorandomness to select the next disjunct formula to expand in the tableau, and also shuffles the order in which the branches are explored, including those introduced by --minimal. The advantage is that some pseudorandom run of HTab on a given input formula could terminate quickly by doing the right choices. Then, this run can be reproduced by setting the pseudorandom seed of HTab with the --seed flag. More information is available by running htab --help.

For all three translations, the implementation has the following particular case:

$$
(\diamond \varphi)_{\emptyset}^{\prime}=\diamond(\varphi)_{\emptyset}^{\prime}
$$

This avoids introducing unnecessary nominals and makes the translated formula more readable. The generated hybrid formula can be seen by using the --showformula flag.

Since the translations are equivalence-preserving, the models built by HTab satisfy the input RC formula. Let us see a few examples. We present the formulas in multi-line way, each line is a conjunct of the whole formula. Also, some new logical connectors are used in the expected way.

Input $\mathscr{M} \mathscr{L}\langle\mathrm{sb}\rangle$ formula:

$$
\begin{aligned}
& \diamond(A \wedge \neg B \wedge \diamond \diamond A) \\
& \diamond(B \wedge \neg A \wedge \diamond \diamond B) \\
& {[\mathrm{sb}](A \rightarrow \square \square \neg A)} \\
& {[\mathrm{sb}](B \rightarrow \square \square \neg B)}
\end{aligned}
$$

Input $\mathscr{M} \mathscr{L}\langle$ gsb $\rangle$ formula:

$$
\begin{aligned}
& \diamond(A \wedge \neg B \wedge \diamond \diamond A) \\
& \diamond(B \wedge \neg A \wedge \diamond \diamond B) \\
& \square \square(C \wedge \square \neg C) \\
& \langle\text { gsb } \square \square \square \square \perp
\end{aligned}
$$

Translated hybrid formula:

$$
\begin{aligned}
& \diamond(A \wedge \neg B \wedge \diamond \diamond A) \\
& \diamond(B \wedge \neg A \wedge \diamond \diamond B) \\
& \downarrow n_{0} \cdot \square\left(\downarrow n_{1} \cdot(\neg A\right. \\
& \vee \downarrow n_{2} \cdot \square\left(\left(n_{1} \wedge n_{2}: n_{0}\right)\right. \\
& \quad \vee \downarrow n_{3} \cdot \square\left(\left(n_{1} \wedge n_{3}: n_{0}\right)\right. \\
& \quad \vee \neg A)))) \\
& \downarrow n_{0} \cdot \square\left(\downarrow n_{1} \cdot(\neg B\right. \\
& \vee \downarrow n_{2} \cdot \square\left(\left(n_{1} \wedge n_{2}: n_{0}\right)\right. \\
& \quad \vee \downarrow n_{3} \cdot \square\left(\left(n_{1} \wedge n_{3}: n_{0}\right)\right. \\
& \quad \vee \neg B))))
\end{aligned}
$$

Translated hybrid formula:

$$
\begin{aligned}
\diamond(A \wedge \neg B \wedge \diamond \diamond A) \\
\diamond(B \wedge \neg A \wedge \diamond \diamond B) \\
\square \square(C \wedge \square \neg C) \\
\downarrow n_{0} . \mathrm{E} \downarrow n_{1} \diamond\left(\downarrow n_{2} .\right. \\
n_{0}:\left(\downarrow n_{3} . \square\left(\left(n_{2} \wedge n_{3}: n_{1}\right)\right.\right. \\
\quad \vee \downarrow n_{4} \cdot \square\left(\left(n_{2} \wedge n_{4}: n_{1}\right)\right. \\
\left.\left.\left.\left.\quad \vee \downarrow n_{5} . \square\left(\left(n_{2} \wedge n_{5}: n_{1}\right)\right)\right)\right)\right)\right)
\end{aligned}
$$

Model found by HTab:

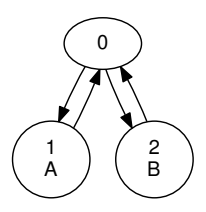

Model found by HTab:

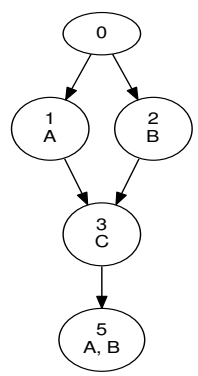




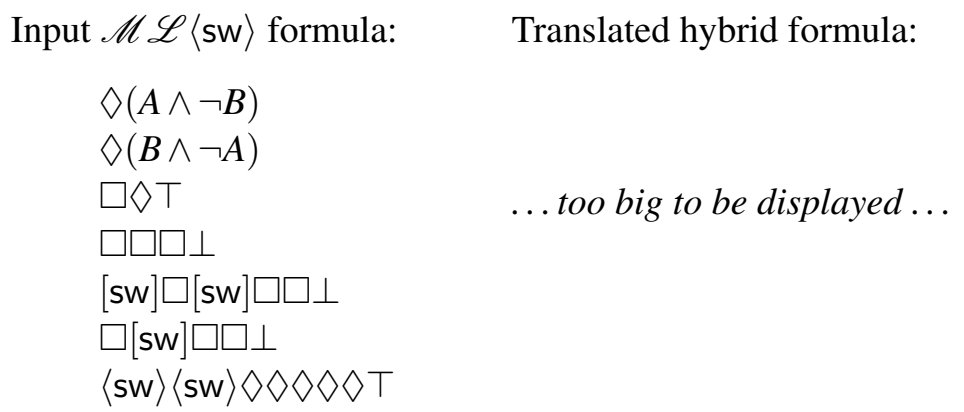

Model found by HTab:

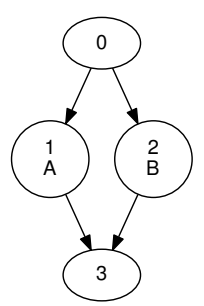

More formulas from the six RC logics are available in the ./ $\mathrm{rc} /$ directory of the HTab source code. This implementation is useful to check the correctness of the translations, just by checking the satisfiable/unsatisfiable output of the prover for known RC formulas. It is also useful for checking that $\mathrm{RC}$ formulas build models in the expected way, such as non-tree or diamond-shaped models.

\section{Decidable Fragments}

Interesting decidable fragments of hybrid logics with binders have been found over time. Such decidable fragments are convenient for our relation-changing logics in the light of the (computable) translations presented in Section 4. First, let us consider restricting the satisfiability problem over certain classes of models. The following logics are known to be decidable over the indicated classes:

- $\mathscr{H} \mathscr{L}(\mathrm{E}, \downarrow)$ over linear frames (i.e., irreflexive, transitive, and trichotomous frames) [17, 24] (this includes $(\mathbb{N},<)$ ),

- $\mathscr{H} \mathscr{L}(\mathrm{E}, \downarrow)$ over models with a single, transitive tree relation [24],

- $\mathscr{H} \mathscr{L}(\mathrm{E}, \downarrow)$ over models with a single, $S 5$, or complete relation [24],

- $\mathscr{H} \mathscr{L}(:, \downarrow)$ over models with a single relation of bounded finite width [14]; as a corollary, also over finite models.

Since the translations preserve equivalence, we get:

Corollary 1. The satisfiability problem for all relation-changing modal logics over linear, transitive trees, S5, and complete frames is decidable.

Corollary 2. The satisfiability problem for local sabotage and local swap logics over models of bounded width is decidable.

Curiously, these results mean that relation-changing modal logics are decidable over certain classes of models, even if the modifications implied by evaluating RC formulas yield models that do not belong to such class. For instance, these two facts are simultaneously true: sabotage logic is decidable on the class of $S 5$ models, and deleting edges in an $S 5$ model can yield a non-S5 model.

Now, let us turn to syntactical definitions of decidable fragments. We recall that local sabotage and local swap can be translated to $\mathscr{H} \mathscr{L}(:, \downarrow)$. Consider formulas of $\mathscr{H} \mathscr{L}(:, \downarrow)$ in negation normal form. $\mathscr{H} \mathscr{L}(:, \downarrow) \backslash \square \downarrow \square$ is the fragment obtained by removing formulas that contain a nesting of $\square, \downarrow$ and again $\square$. This fragment is decidable [14].

Our translations do use the $\downarrow$ binder in many places, but we can make them a little more economical in that sense, at the expense of losing succinctness. 
Take the following case for $\mathscr{M} \mathscr{L}(\langle\mathrm{sw}\rangle)$ :

$$
(\diamond \varphi)_{S}^{\prime}=\downarrow n \cdot \diamond\left(\neg \text { belongs }(n, S) \wedge(\varphi)_{S}^{\prime}\right) .
$$

Instead of using the down-arrow binder and later ensuring that we did not take a deleted edge by using $\neg$ belongs $(n, S)$, we can do the following. For all pairs of nominals $(x, y) \in S$, the current state $w$ satisfies one combination of the truth values of the nominals $x$. Let $X$ be the set of true nominals $x$ at $w$. Then, $(\varphi)_{S}^{\prime}$ should be true at some accessible state $v$ that should not satisfy any of the corresponding $y$ nominals for all $x \in X$.

Then, the translation becomes:

$$
(\diamond \varphi)_{S}^{\prime}=\bigvee_{X \subseteq \text { fst }(\mathrm{S})}\left(\bigwedge_{x \in X} x \wedge \bigwedge_{x \notin X} \neg x \wedge \diamond\left(\bigwedge_{y \in \operatorname{snd}(S, X)} \neg y \wedge(\varphi)_{S}^{\prime}\right)\right)
$$

where fst $(S)=\{x \mid(x, y) \in S\}$ and $\operatorname{snd}(S, X)=\{y \mid(x, y) \in S, x \in X\}$.

In the case of $\mathscr{M} \mathscr{L}(\langle\mathrm{sw}\rangle)$ we can do the same. We recall that the case introduced in Section 4 was:

$$
(\diamond \varphi)_{S}^{\prime}=\left(\downarrow n . \diamond\left(\neg \operatorname{belongs}(n, S) \wedge(\varphi)_{S}^{\prime}\right)\right) \vee \operatorname{isSat}\left(S^{-1},(\varphi)_{S}^{\prime}\right) .
$$

Here the isSat $\left(S^{-1},(\varphi)_{S}^{\prime}\right)$ disjunct does not use the $\downarrow$ binder, while the first disjunct is similar to the case of local sabotage, and can be replaced accordingly:

$$
\begin{aligned}
& (\diamond \varphi)_{S}^{\prime}=\quad \bigvee_{X \subseteq \text { fst }(S)}\left(\bigwedge_{x \in X} x \wedge \bigwedge_{x \notin X} \neg x \wedge \diamond\left(\bigwedge_{y \in \operatorname{snd}(S, X)} \neg y \wedge(\varphi)_{S}^{\prime}\right)\right) \\
& \vee \text { isSat }\left(S^{-1},(\varphi)_{S}^{\prime}\right) \text {. }
\end{aligned}
$$

Let be either $\langle s b\rangle$ or $\langle s w\rangle$ and $\boldsymbol{D}$ be either [sb] or [sw]. The following patterns in RC formulas provoke the following patterns in the hybrid formula produced by the translations:

\begin{tabular}{|c|c|}
\hline RC pattern & Produced pattern \\
\hline$\square$ & $\square$ \\
\hline$\bullet$ & $\downarrow$ \\
\hline$\square$ & $\downarrow \square \downarrow$ \\
\hline
\end{tabular}

By considering these new versions of the translations, and by taking into account the syntactic decidable fragment of $\mathscr{H} \mathscr{L}(:, \downarrow)$ mentioned above, we can establish the following result:

Corollary 3. The following fragments are decidable on the class of all relational models:

- $\mathscr{M} \mathscr{L}(\langle\mathrm{sb}\rangle) \backslash\{\mathbf{\square}, \mathbf{\square} \square, \square \mathbf{\square}, \square \vee \square\}$

- $\mathscr{M} \mathscr{L}(\langle\mathrm{sw}\rangle) \backslash\{\mathbf{\square}, \mathbf{\square} \square, \square \mathbf{\square}, \square \vee \square\}$

where $\square$ is either $\square$ or

\section{Comparing Expressive Power}

We have introduced translations for the six relation-changing modal logics from Section 2 into hybrid logic. In some cases (for the local version of swap and sabotage), the obtained formulas fall into the fragment $\mathscr{H} \mathscr{L}(:, \downarrow)$. On the other hand, for encoding the rest of the logics we need also to use the universal modality E. An interesting question is whether we can obtain translations from hybrid to relation-changing logics, i.e., if some of the relation-changing logics considered in this article are as expressive as some hybrid logic. Let us define first, the expressive power comparisons we will use. 
Definition $7\left(\mathscr{L} \leq \mathscr{L}^{\prime}\right)$. We say that $\mathscr{L}^{\prime}$ is at least as expressive as $\mathscr{L}$ (notation $\mathscr{L} \leq \mathscr{L}^{\prime}$ ) if there is a function $\operatorname{Tr}$ between formulas of $\mathscr{L}$ and $\mathscr{L}^{\prime}$ such that for every model $\mathscr{M}$ and every formula $\varphi$ of $\mathscr{L}$ we have that

$$
\mathscr{M} \models \mathscr{L} \varphi \text { iff } \mathscr{M} \models \mathscr{L}^{\prime} \operatorname{Tr}(\varphi) .
$$

$\mathscr{M}$ is seen as a model of $\mathscr{L}$ on the left and as a model of $\mathscr{L}^{\prime}$ on the right, and we use in each case the appropriate semantic relation $\models \mathscr{L}$ or $\models \mathscr{L}^{\prime}$ as required.

$\mathscr{L}^{\prime}$ is strictly more expressive than $\mathscr{L}\left(\mathscr{L}<\mathscr{L}^{\prime}\right)$ if $\mathscr{L} \leq \mathscr{L}^{\prime}$ but not $\mathscr{L}^{\prime} \leq \mathscr{L}$. Finally, we say that $\mathscr{L}$ and $\mathscr{L}^{\prime}$ are incomparable if $\mathscr{L} \$ \mathscr{L}^{\prime}$ and $\mathscr{L}^{\prime} \not \mathscr{L}$.

In $[5,7,16,8]$ we discussed the expressive power of relation-changing modal logics by introducing their corresponding notions of bisimulations and using them to compare the logics among each other. We concluded that they are all incomparable in expressive power. ${ }^{1}$ As a consequence, we conclude that it is not possible that two of them capture the same fragment of hybrid logic. In fact, we will prove that all the relation-changing logics considered here are strictly less expressive than the corresponding hybrid logic in which they are translated.

Theorem 4. Let ${ }_{1} \in\{\langle\mathrm{sb}\rangle,\langle\mathrm{sw}\rangle\}$, we have $\mathscr{M} \mathscr{L}\left({ }_{1}\right)<\mathscr{H} \mathscr{L}(:, \downarrow)$. For $\boldsymbol{}_{2} \in\{\langle\mathrm{gsb}\rangle,\langle\mathrm{gsw}\rangle,\langle\mathrm{br}\rangle,\langle\mathrm{gbr}\rangle\}$, we have $\mathscr{M} \mathscr{L}\left(\vee_{2}\right)<\mathscr{H} \mathscr{L}(\mathrm{E}, \downarrow)$.

Proof. For any of the logics mentioned above, we have translations into the corresponding hybrid logic. Now we need to prove that these translations do not cover their entire target language (modulo equivalence). In order to do that, we provide bisimilar models for relation-changing modal logics which can be distinguished by some hybrid formula. In Figure 1, we show two pairs of models already introduced in [8] that cover all possibilities of bisimilarity.

\begin{tabular}{|l|l|l|}
\hline $\mathscr{M}, w$ & $\mathscr{M}^{\prime}, w^{\prime}$ & Bisimilar for \\
\hline \hline$w$ & & $\mathscr{M} \mathscr{L}(\langle\mathrm{sw}\rangle)$ \\
& & $\mathscr{M} \mathscr{L}(\langle\mathrm{b}\rangle)$ \\
$w^{\prime}$ & $\mathscr{M} \mathscr{L}(\langle\mathrm{gsw}\rangle)$ \\
& $\mathscr{M} \mathscr{L}(\langle\mathrm{gbr}\rangle)$ \\
\hline$w$ & $\vdots$ & $\mathscr{M} \mathscr{L}(\langle\mathrm{sb}\rangle)$ \\
& $\vdots$ & $\mathscr{M} \mathscr{L}(\langle\mathrm{gsb}\rangle)$ \\
\hline
\end{tabular}

Figure 1: Bisimilar models

The two models in the first row can be distinguished by the formula $\downarrow n$. $\square n$, which establishes that the only successor of the evaluation point is itself. This formula is true at $\mathscr{M}, w$ and false at $\mathscr{M}^{\prime}, w^{\prime}$. Models in the second row can be distinguished by the formula $\downarrow n . \vee \downarrow m . n: \diamond \diamond m$, which says that from the evaluation point it is possible to arrive to the same state in one or two steps. This is true at $\mathscr{M}, w$ but false at $\mathscr{M}^{\prime}, w^{\prime}$.

Notice that both hybrid formulas we introduced above belong to the fragment $\mathscr{H} \mathscr{L}(:, \downarrow)$, i.e., it was not necessary to use the $\mathrm{E}$ operator. This means that even though we use $\mathrm{E}$ in some of the translations (and we strongly believe that it is essential for some encodings) there are fragments of $\mathscr{H} \mathscr{L}(:, \downarrow)$ that cannot be captured by relation-changing modal logics.

\footnotetext{
${ }^{1}$ Except for the local and global swap operators, which is still open in one direction.
} 


\section{Final Remarks}

In this article, we introduced equivalence-preserving translations from six logics we named relationchanging to a very expressive hybrid logic. We considered three kinds of modifications: deleting, adding, and swapping edges, that can be performed both globally (anywhere in the model) and locally (modifying adjacent edges from the evaluation point). On the other hand, hybrid logic has operators to rename states in a model with some particular atomic symbols named nominals. We use the down-arrow operator $\downarrow$ to name pairs of states that represent modified states. In this way, we keep track of the evolution of a model.

It is known that the hybrid logic $\mathscr{H} \mathscr{L}(\mathrm{E}, \downarrow)$ has the same expressive power as $\mathscr{F} \mathscr{O} \mathscr{L}$, and we introduced standard translations from relation-changing logics to $\mathscr{F} \mathscr{O} \mathscr{L}$ in [8]. However, by giving explicit translations to hybrid logic we can benefit from its decidable fragments to find decidable fragments of relation-changing modal logics. Also, these translations are useful to analyze expressive power. We showed that the six logics we considered are strictly less expressive than $\mathscr{H} \mathscr{L}(\mathrm{E}, \downarrow)$. In fact, despite we used the modality $E$ in some translations, all relation-changing logics we considered here cannot capture the full fragment $\mathscr{H} \mathscr{L}(:, \downarrow)$ (which is less expressive than $\mathscr{H} \mathscr{L}(\mathrm{E}, \downarrow)$ ). In summary, we learned that relation-changing modal logics are languages that enable to talk directly and succinctly about distinct kinds of model modifications, but with a little effort they can be simulated by hybrid logics.

Translating to $\mathscr{H} \mathscr{L}(\mathrm{E}, \downarrow)$ also enabled us to easily obtain an implementation for relation-changing modal logics, by extending the hybrid logic theorem prover HTab. Satisfiability checking and model building can thus be automated and were useful to empirically verify our translations on concrete cases. However, we did not implement the changes of Section 6. Indeed, although in some cases they provide a way to avoid using the down-arrow binder in the translations (which is a source of undecidability for hybrid logic), they also make the generated formulas impractically bigger.

We studied six relation-changing modal logics with the goal of covering a sufficiently varied sample of alternatives. Clearly, other operators could have been included in this exploration, and actually some alternative choices have been already investigated in the literature, e.g., the adjacent sabotage operator discussed in [23], or the more generic approach investigated in [8].

Even though relation-changing modal logics have been extensively investigated [16, 8], there are still many interesting questions to be answered. The hybrid perspective we introduced in this article, as well as in [6], gives us a new way to think of the relation-changing framework. As an example, we can use hybridization techniques (a very standard technique in modal logic [11]) to find complete axiomatizations or compute interpolants for relation-changing modal logics.

Acknowledgments: This work was partially supported by grant ANPCyT-PICT-2013-2011, DFG grant LU 1417/2-1, and the Laboratoire International Associé "INFINIS".

\section{References}

[1] C. Areces, P. Blackburn \& M. Marx (1999): A Road-map on Complexity for Hybrid Logics. In J. Flum \& M. Rodríguez-Artalejo, editors: Computer Science Logic, Lecture Notes in Computer Science 1683, Springer, Madrid, Spain, pp. 307-321, doi:10.1007/3-540-48168-0_22.

[2] C. Areces \& B. ten Cate (2007): Hybrid Logics. In P. Blackburn, F. Wolter \& J. van Benthem, editors: Handbook of Modal Logic, Elsevier, pp. 821-868, doi:10.1016/s1570-2464(07)80017-6.

[3] C. Areces, H. van Ditmarsch, R. Fervari \& F. Schwarzentruber (2014): Logics with Copy and Remove. In: Logic, Language, Information, and Computation, Lecture Notes in Computer Science 8652, Springer, pp. 51-65, doi:10.1007/978-3-662-44145-9_4. 
[4] C. Areces, H. van Ditmarsch, R. Fervari \& F. Schwarzentruber (2015): The Modal Logic of Copy and Remove. To Appear in Information and Computation, special issue of WoLLIC 2014.

[5] C. Areces, R. Fervari \& G. Hoffmann (2012): Moving Arrows and Four Model Checking Results. In: Logic, Language, Information and Computation, Lecture Notes in Computer Science 7456, Springer, pp. 142-153, doi:10.1007/978-3-642-32621-9_11.

[6] C. Areces, R. Fervari \& G. Hoffmann (2013): Tableaux for Relation-Changing Modal Logics. In: Frontiers of Combining Systems, Lecture Notes in Computer Science 8152, pp. 263-278, doi:10.1007/978-3-642-408854_19.

[7] C. Areces, R. Fervari \& G. Hoffmann (2014): Swap Logic. Logic Journal of the IGPL 22(2), pp. 309-332, doi:10.1093/jigpal/jzt030.

[8] C. Areces, R. Fervari \& G. Hoffmann (2015): Relation-Changing Modal Operators. Logic Journal of the IGPL 23(4), pp. 601-627, doi:10.1093/jigpal/jzv020.

[9] J. van Benthem (2005): An Essay on Sabotage and Obstruction. In: Mechanizing Mathematical Reasoning, pp. 268-276, doi:10.1007/978-3-540-32254-2_16.

[10] P. Blackburn \& J. van Benthem (2007): Modal Logic: A Semantic Perspective. In: Handbook of Modal Logic, Elsevier, pp. 1-84, doi:10.1016/s1570-2464(07)80004-8.

[11] P. Blackburn, M. de Rijke \& Y. Venema (2001): Modal Logic. Cambridge Tracts in Theoretical Computer Science, Cambridge University Press, doi:10.1017/CBO9781107050884.

[12] P. Blackburn \& J. Seligman (1995): Hybrid Languages. Journal of Logic, Language and Information 4(3), pp. 251-272, doi:10.1007/BF01049415.

[13] B. ten Cate (2005): Model theory for extended modal languages. Ph.D. thesis, University of Amsterdam. ILLC Dissertation Series DS-2005-01.

[14] B. ten Cate \& M. Franceschet (2005): On the complexity of hybrid logics with binders. Lecture Notes in Computer Science 3634, Springer Verlag, pp. 339-354, doi:10.1007/11538363_24.

[15] H. van Ditmarsch, W. van der Hoek \& B. Kooi (2007): Dynamic Epistemic Logic. Synthese Library, Springer, doi:10.1007/978-1-4020-5839-4.

[16] R. Fervari (2014): Relation-Changing Modal Logics. Ph.D. thesis, Universidad Nacional de Córdoba, Argentina.

[17] M. Franceschet, M. de Rijke \& B. Schlingloff (2003): Hybrid Logics on Linear Structures: Expressivity and Complexity. In: TIME-ICTL 2003, Cairns, Queensland, Australia, pp. 166-173, doi:10.1109/time.2003.1214893.

[18] N. Gierasimczuk, L. Kurzen \& F. R. Velázquez-Quesada (2009): Learning and Teaching as a Game: A Sabotage Approach. In Xiangdong He, John F. Horty \& Eric Pacuit, editors: LORI, Lecture Notes in Computer Science 5834, Springer, pp. 119-132, doi:10.1007/978-3-642-04893-7_10.

[19] V. Goranko \& S. Passy (1992): Using the Universal Modality: Gains and Questions. Journal of Logic and Computation 2(1), pp. 5-30, doi:10.1093/logcom/2.1.5.

[20] G. Hoffmann \& C. Areces (2009): HTab: A Terminating Tableaux System for Hybrid Logic. Electronic Notes in Theoretical Computer Science 231, pp. 3-19, doi:10.1016/j.entcs.2009.02.026.

[21] C. Löding \& P. Rohde (2003): Model Checking and Satisfiability for Sabotage Modal Logic. Lecture Notes in Computer Science 2914, pp. 302-313, doi:10.1007/978-3-540-24597-1_26.

[22] M. Martel (2015): On the Undecidability of Relation-Changing Logics. Master's thesis, Universidad Nacional de Río Cuarto, Argentina.

[23] P. Rohde (2006): On games and logics over dynamically changing structures. Ph.D. thesis, RWTH Aachen.

[24] T. Schneider (2007): The Complexity of Hybrid Logics over Restricted Frame Classes. Ph.D. thesis, University of Jena.

[25] E. Spaan (1993): Complexity of modal logics. Ph.D. thesis, ILLC, University of Amsterdam. 